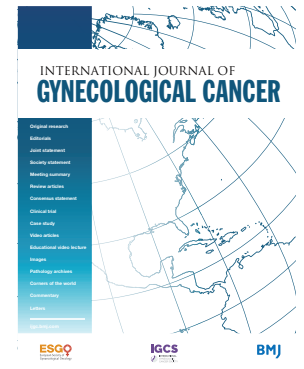

Correspondence to Dr Gloria Salvo, Department of Gynecologic Oncology and Reproductive Medicine, The University of Texas MD Anderson Cancer Center, Houston, TX 77030, USA; gsalvo@mdanderson.org

Accepted 7 May 2021

\title{
Early-stage, high-grade neuroendocrine cervical carcinoma
}

\author{
Presenter: \\ Gloria Salvo \\ Department of Gynecologic Oncology and Reproductive Medicine, The University of Texas MD \\ Anderson Cancer Center, Houston, Texas, USA \\ Pathologist: \\ Preetha Ramalingam \\ Department of Pathology, University of Texas MD Anderson Cancer Center, Houston, Texas, USA \\ Radiologist: \\ Priya Bhosale \\ Department of Diagnostic Imaging, University of Texas MD Anderson Cancer Center, Houston, Texas, \\ USA \\ Department of Radiology, University of Texas MD Anderson Cancer Center Division of Cancer \\ Medicine, Houston, Texas, USA \\ Discussant: \\ Michael Frumovitz \\ Department of Gynecologic Oncology and Reproductive Medicine, The University of Texas MD \\ Anderson Cancer Center, Houston, Texas, USA
}

\section{CASE PRESENTATION}

A 37-year-old woman presented to an outside institution in November 2015 with at least a 1-year history of heavy vaginal bleeding, anemia, and chronic pelvic pain. She had a past medical history of arrythmia, asthma, seizures, and obesity (body mass index $38.9 \mathrm{~kg} / \mathrm{m}^{2}$ ). Her surgical history included a C-section 5 years prior to presentation and a hysteroscopy with bilateral tubal cannulation in January 2014. The patient was gravida 5, para 4 and had a long history of cervical dysplasia. The patient was diagnosed with a low-grade intraepithelial lesion in 2005 (no human papillomavirus (HPV) test was performed), and with cervical intraepithelial neoplasia grade 2 on a loop electrosurgical excision procedure in 2006. In 2012, she had a negative Pap smear with high-risk HPV (unknown type). Her family history was significant with a maternal grandmother with unspecified gynecologic malignancy.

At consultation at the outside facility the patient requested a hysterectomy to address both the bleeding and pain as she related both to the tubal cannulation procedure in 2014. To evaluate the vaginal bleeding a Pap smear was performed which revealed atypical squamous cells of undetermined significance and positive HPV DNA test (16/18+). A bimanual pelvic examination showed a multiparous cervix. The cervix was enlarged with no exophytic mass seen on speculum evaluation, although visualization was difficult due to body habitus and discomfort. With cytology results, a colposcopic evaluation was done with no acetowhite lesions. Due to the absence of a visible lesion, multiple random cervical biopsies and endocervical curettage were carried out.

\section{Dr Ramalingam}

The endocervical curettage showed a highgrade neuroendocrine carcinoma, small cell type (Figure 1A). The tumor was composed of cells with high nuclear-to-cytoplasmic ratio, molding, and crush artifact. The biopsy was not well oriented precluding accurate depth of invasion, but the tumor involved the full thickness of the tissue (at least $1.3 \mathrm{~mm}$ ). Immunohistochemical stains showed the tumor cells to be positive for low molecular weight cytokeratin, p16 (diffuse block-like staining), chromogranin, synaptophysin, and CD56, while negative for CK5 and p63 (Figure 1B,C). The morphology and immunophenotype were diagnostic of high-grade neuroendocrine carcinoma, small cell type.

\section{Dr Bhosale}

A pelvic MRI scan with and without contrast showed a $2 \times 2 \times 1.2 \mathrm{~cm}$ enhancing mass in the posterior tip of the cervix with intermediate T2 signal and associated restricted effusion corresponding to the known primary malignancy. There was no vaginal extension or parametrial invasion. The ovaries were unremarkable. 


\section{Case study}
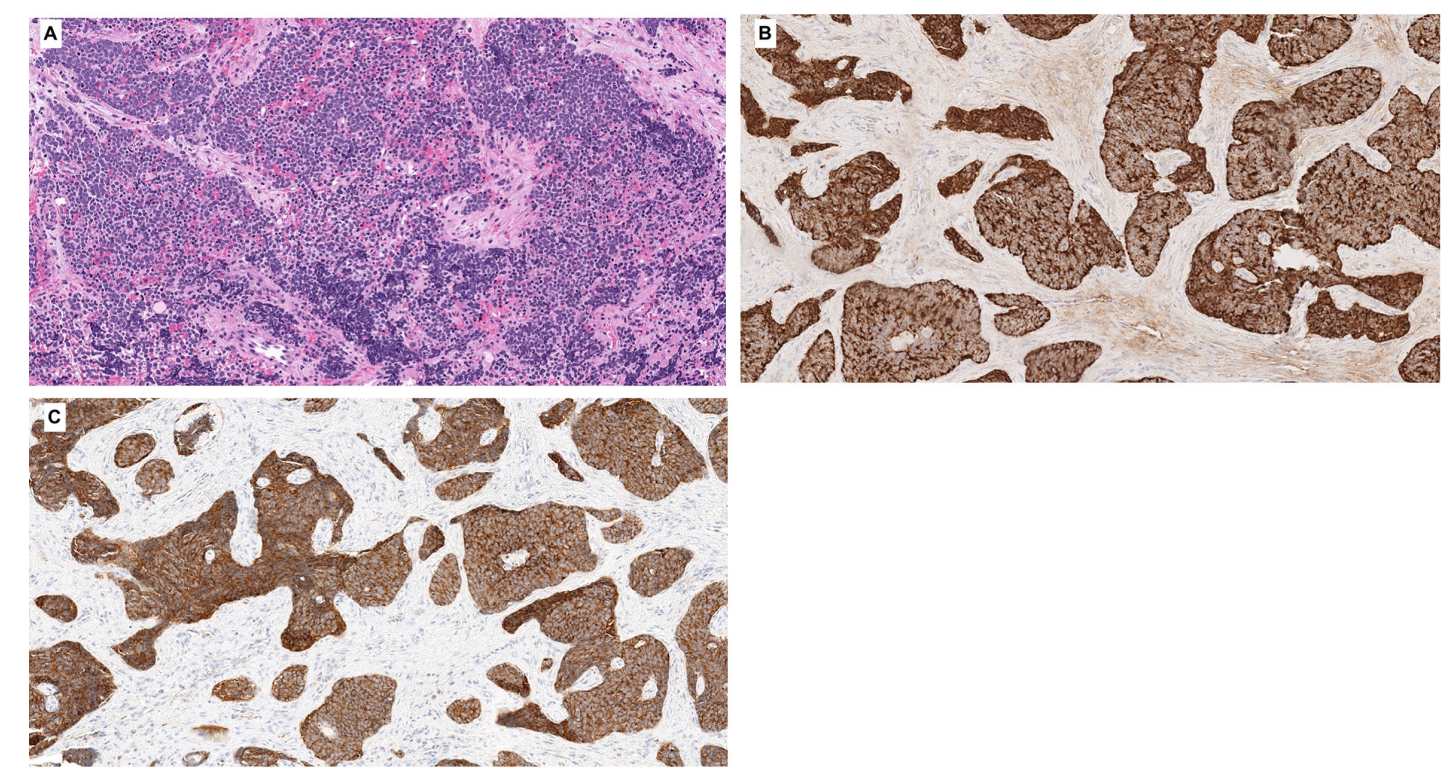

Figure 1 Tumor showing high nuclear-cytoplasmic ratio (A) with nuclear molding and crush artifact (Azzopardi effect). Tumor cells are positive for chromogranin (B) and synaptophysin (C).

No pelvic or para-aortic nodal disease and no evidence of distant or metastatic disease (Figure 2A,B).

\section{Dr Frumovitz}

Based on this diagnosis, what would you consider ideal recommendations regarding imaging evaluation of this patient?

Based on the diagnosis of high-grade neuroendocrine carcinoma, small cell type, with a $2 \mathrm{~cm}$ lesion on cervical imaging, we would start with a PET scan to evaluate metastatic disease. Assuming the imaging shows disease limited to the cervix, we would not perform a brain MRI scan as metastatic disease to the brain without disease spread first to the liver and/or lung in an asymptomatic patient is exceedingly rare. In the past, some have advocated prophylactic cranial irradiation as is frequently done for small cell lung cancer; however, this is not indicated for women with small cell cervical cancer and should not be performed.

The patient had a FIG0 2018 stage IB1 cervical tumor and in December 2015 she underwent a laparoscopic radical hysterectomy
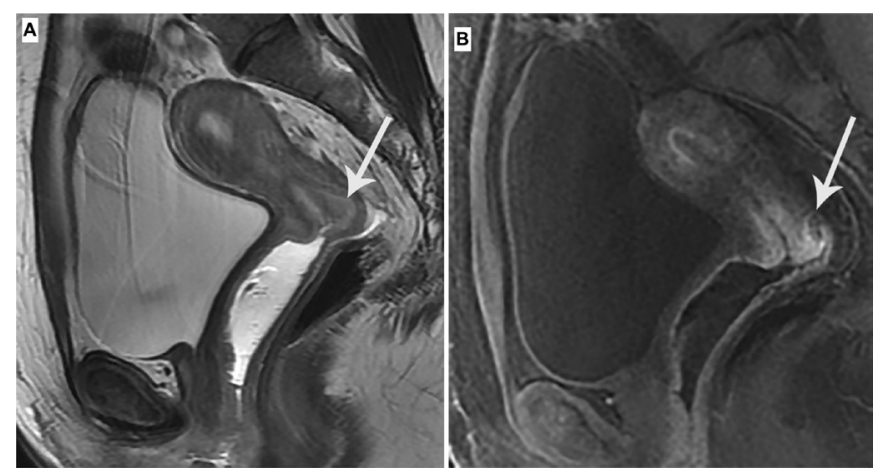

Figure 2 Pelvic MRI. sagittal T2 sequence (A) shows a mass in the posterior cervix (arrow). Sagittal post-contrast T1-weighted sequence $(B)$ shows a mass in the posterior cervix (arrow). with bilateral salpingo-oophorectomy and bilateral pelvic lymph node dissection at an outside facility.

\section{Dr Frumovitz}

a. Can you comment on the role of lymphadenectomy versus sentinel lymph node mapping in the setting of neuroendocrine carcinoma of the cervix?

b. Are there any data in support of ovarian preservation in the setting of early-stage cervical neuroendocrine carcinoma?

Our standard treatment for a patient with stage IB1 high-grade neuroendocrine carcinoma of the cervix would be to start with an open radical hysterectomy and sentinel lymph node biopsies with either bilateral salpingo-oophorectomy or bilateral salpingectomy with ovarian transposition. Although women with neuroendocrine carcinoma were excluded from the Laparoscopic Approach to Cervical Cancer (LACC) trial, we do not recommend a minimally invasive approach for performing the radical hysterectomy.

As patients with clinical stage I, high-grade neuroendocrine carcinoma of the cervix have a high likelihood of metastatic disease to the pelvic lymph nodes, assessment of these regional nodes should be performed. It is unknown if resecting microscopically positive nodes is therapeutic or just prognostic, especially in light of the fact that most patients with high-grade neuroendocrine carcinoma of the cervix will get pelvic radiation, even when nodes are pathologically negative. That said, we recommend nodal assessment with either complete pelvic lymphadenectomy or with sentinel lymph node biopsy. We favor sentinel lymph node biopsy as it probably reduces lower extremity lymphedema compared with complete lymphadenectomy when combined with pelvic radiation.

With the average age of women with high-grade neuroendocrine cervical cancer being younger than for those with squamous cell or adenocarcinoma of the cervix, many patients, such as this one, will be premenopausal and concerned about the short- and longterm effects and surgical menopause. As mentioned, most patients with stage I disease will receive adjuvant pelvic radiation, and thus 
leaving ovaries in the pelvis does not prevent ovarian failure. In young patients we have performed ovarian transposition if the ovaries appear grossly normal. The risk of isolated microscopic disease to the ovaries is exceedingly low so we are not concerned about potentially moving a diseased ovary out of the radiation field.

\section{Dr Ramalingam}

The radical hysterectomy specimen showed high-grade neuroendocrine carcinoma small cell type. Anterior cervix remarkable for a $1.9 \mathrm{~cm} \times 1.8 \mathrm{~cm}$ cylindrical mass that compromises the lower uterine cervix. Tumor depth of invasion was 11 of $16 \mathrm{~mm}$ cervical thickness. Unequivocal lymphovascular invasion was not identified. All 15 pelvic nodes, bilateral ovaries, and vaginal and parametrial margins were negative for tumor. There was no vaginal or parametrial compromise.

Post-operative course was complicated by pelvic abscess requiring re-admission for $I V$ antibiotics and Jackson-Pratt drain placement. She was discharged with flagyl and doxycycline to complete a 14-day course of antibiotics. The drain was removed after 20 days. A CT scan showed complete resolution of the abscess.

After resolution of the pelvic abscess, the patient underwent six cycles of cisplatin $\left(60 \mathrm{mg} / \mathrm{m}^{2}\right.$ on day 1 every 3 weeks) and etoposide $\left(100 \mathrm{mg} / \mathrm{m}^{2}\right.$ on days $1-3$ every 3 weeks) followed by external beam radiation therapy (45Gy in 25 fractions) and high-dose brachytherapy (6Gy in two fractions) to a total dose of 51 Gy. No concurrent chemotherapy was given with the radiation. At completion of primary treatment, a PET-CT scan (September 2016) showed no evidence of disease.

\section{Dr Frumovitz}

\section{a. Can you comment on the recommendation for combined radical hysterectomy followed by chemotherapy and subsequent pelvic radiation?}

b. Is there a role for simple hysterectomy given that a patient will receive radiation therapy regardless of findings on final pathology?

c.ls there consideration of an alternative chemotherapy regimen when considering adjuvant treatment?

Our current practice for patients with clinical and radiologic stage I tumors $<4 \mathrm{~cm}$ in size is open radical hysterectomy with sentinel lymph node biopsies and bilateral salpingo-oophorectomy or bilateral salpingectomy with ovarian transposition. If pathology confirms cervix only disease, we follow surgery with chemoradiation (pelvic field) with cisplatin and etoposide $\times$ two cycles and then an additional four cycles of cisplatin and etoposide after completing radiation. Most patients are able to tolerate the combination with granulocyte colony stimulating factors support. For patients receiving chemoradiation, we reduce the dosage of etoposide from $120 \mathrm{mg} /$ $\mathrm{m}^{2}$ to $100 \mathrm{mg} / \mathrm{m}^{2}$. We encourage patients to have at least five cycles of chemotherapy as multiple retrospective studies have shown that women who have five or more cycles cycles have better outcomes than those who have fewer than five cycles.

Because the risk of pelvic recurrence is high, we feel strongly that radiation should be part of post-operative adjuvant therapy. Multiple studies have shown that adding radiation significantly decreases pelvic recurrences after radical hysterectomy; however, interestingly, it has not been shown to improve survival. It is unknown whether this is because most studies are too small to show a statistical difference or if the high risk of distant recurrence negates the advantage of reducing pelvic recurrences. At this point we believe it is the former and so continue to recommend radiation post-operatively. That said, we are continually re-evaluating this approach and may one day decide that radiation does not add benefit.

Because our current standard is post-operative radiation therapy for almost all patients, even those with pathologic stage IB1/IB2 disease, we have been contemplating the role of radical hysterectomy in these patients. We recently showed that $10 \%$ of patients with clinical/radiologic stage I tumors $<4 \mathrm{~cm}$ will have parametrial involvement, and in all of these patients the tumor burden in the parametrium microscopic. As all of our patients currently receive post-operative radiation, we have discussed offering patients a simple, rather than radical, hysterectomy, with sentinel lymph node mapping. Our approach at MD Anderson is still a radical surgery but we are starting to question if this approach might be excessive.

Nine months (June 2017) after completing primary treatment the patient returned to clinic complaining of right-side abdominal pain and vomiting. On physical examination her blood pressure and heart rate were within normal limits. She reported a pain score of 5/10, Eastern Cooperative Oncology Group status 1. General appearance was of a well-appearing individual in no apparent distress. Head and neck examination was unremarkable, lungs clear to auscultation, and cardiovascular examination was within normal limits. Abdominal examination was negative. On bimanual examination no nodularity, mass, or tenderness was noticed. Rectal examination confirms this finding.

\section{Dr Bhosale}

A PET-CT scan showed a new ill-defined hypodense and hypermetabolic lesion in segment 7 of the liver, measuring approximately $1.2 \times 2.2 \mathrm{~cm}$ with standardized uptake value (SUV) 8.6. Additional hypermetabolic foci were noted in segments 5 and 6 . Multiple new hypermetabolic peritoneal nodules were also identified. Additional subcentimeter peritoneal nodules were noted without significant fluorodeoxyglucose (FDG) uptake but also suspicious for carcinoma. New hypermetabolism in ill-defined areas of stranding in the pelvis, possibly reflecting local tumor recurrence, was also noted. New hypermetabolic left and right pelvic sidewall adenopathy was also seen (Figure 3).

The patient underwent a left pelvic mass biopsy confirming recurrent, metastatic small cell carcinoma. A CT scan of the brain showed no metastatic disease. A solid tumor genomic test was performed using surgical specimen tissue, with no somatic mutations identified. A DNA microsatellite instability analyses (PCRbased method evaluating a panel of seven microsatellite markers BAT25, BAT26, BAT40, D2S123, D5S346, D17S250, and TGFBR2) showed a microsatellite stable tumor. PD-L1 immunohistochemistry showed a combined positive score $<1$. Treatment was started with topotecan $\left(0.75 \mathrm{mg} / \mathrm{m}^{2}\right.$ on days $\left.1-3\right)$, paclitaxel $\left(175 \mathrm{mg} / \mathrm{m}^{2}\right.$ on day 1), and bevacizumab (15 mg/kg on day 1$)$ every 21 days. A PET-CT scan was ordered every three cycles showing complete response after cycle 9 . The patient performance status was 0 , but chemotherapy was exceedingly difficult to undertake, and she required inpatient admission for all cycles. After discussing different options, such as continuing the three-drug regimen versus paclitaxel and 


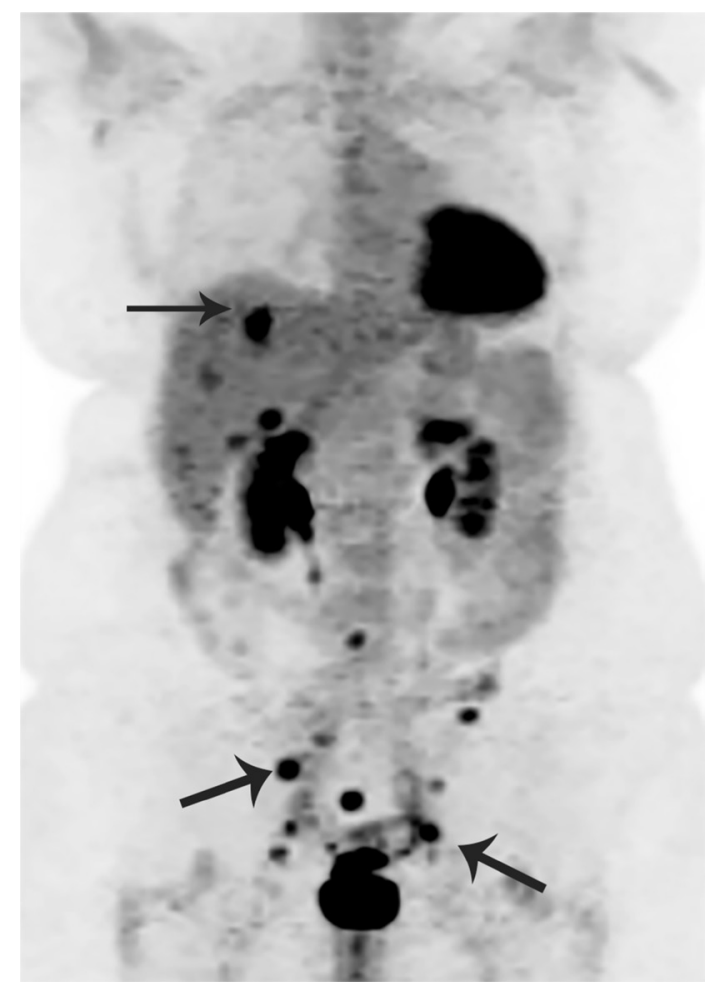

Figure 3 Maximum intensity projection image of the PET/ CT scan performed in June 2017 showing metastatic disease in the liver (top arrow) and metastatic adenopathy (arrows).

bevacizumab only versus bevacizumab maintenance versus observation the decision was made to switch to paclitaxel and bevacizumab (bevacizumab was held for two cycles due to hypertension). After three cycles she continue to have complete response. After a total of six additional cycles of paclitaxel and bevacizumab a PET scan showed recurrent disease in the pelvic lymph nodes, mesenteric nodes, and groin.

\section{Dr Frumovitz}

a.What is the evidence in the literature as to the best systemic therapy for recurrent cervical neuroendocrine carcinoma?

\section{b. Is there any evidence for use of targeted therapy in the setting of neuroendocrine cervical carcinoma in the recurrent or advanced setting?}

Due to the rarity of this disease, there are no prospective studies on any treatments for women with high-grade neuroendocrine carcinoma of the cervix. Much of the chemotherapy employed for women with this malignancy is adopted from the treatment of small cell lung cancer and high-grade neuroendocrine carcinomas from other sites such as the gastrointestinal system. For example, the use of cisplatin and etoposide in the upfront setting comes from similar treatment algorithms for small cell lung cancer. Beyond this chemotherapy regimen in patients with newly diagnosed disease employed by most providers, however, there were no standard recommendations for women with recurrent disease.

In 2013 we started prescribing the three-drug regimen topotecan, paclitaxel, and bevacizumab for treatment of recurrent high-grade neuroendocrine carcinoma of the cervix. The rationale was based on multiple factors. First, topotecan and paclitaxel are commonly used as single agents in treating patients with small cell lung cancer. Second, small cell neuroendocrine carcinoma cells stained positive for the vascular endothelial growth factor receptor about $95 \%$ of the time. Third, the three-drug regimen was used in GOG240 trial without excessive toxicity in women with recurrent squamous or adenocarcinoma, most of whom had been treated with definitive chemoradiation so we felt the combination would be safe in patients with recurrent cervical neuroendocrine carcinoma. Finally, as all three drugs were FDA approved for treating cervical cancer, we expected that we would be able to get them approved by most insurers (and we were correct). This regimen is very active in patients with recurrent neuroendocrine carcinoma, and we have seen multiple patients receiving treatment for $>2$ years. This regimen has become our standard for recurrent disease and has become known among neuroendocrine patients as 'The Texas Cocktail.'

When patients progress on topotecan, paclitaxel, and bevacizumab, there are minimal options. We have given patients single-agent temozolomide as they do for small cell lung cancer as well as temozolomide and capecitabine, which is frequently used in recurrent gastrointestinal neuroendocrine carcinomas. Neither of these regimens, unfortunately, has shown much activity. Likewise, we have seen minimal response to single agent PD-1/PD-L1 inhibitors. If possible, we actively seek clinical trials for women with recurrent disease. Most of these are based on molecular testing, so we recommend strongly that molecular testing is performed at first recurrence to inform future trial options.

\section{Dr Bhosale}

A PET/CT scan (June 2018) demonstrated two FDG-avid left lower quadrant implants. There was focal ill-defined FDG uptake in the right hepatic lobe at the dome measuring SUV max 5.9. There was interval development of a small but hypermetabolic mesenteric node in the left lower quadrant, measuring $6 \mathrm{~mm}$ and SUV max 7.1. There was also another enlarged hypermetabolic nodule/node in the left lower abdomen, measuring $1.5 \mathrm{~cm}$ and SUV max 20.6. Also, a new right inguinal node measured approximately $0.7 \mathrm{~cm}$ and SUV $\max 6.1$ (Figure 4A).

The decision was made to restart topotecan $\left(0.75 \mathrm{mg} / \mathrm{m}^{2}\right.$ on days $1-3)$, paclitaxel $\left(175 \mathrm{mg} / \mathrm{m}^{2}\right.$ on day 1$)$, and bevacizumab ( $15 \mathrm{mg} / \mathrm{kg}$ on day 1 ) every 21 days. Cycles $4-7$ were complicated by colitis and later cellulitis/erysipelas, and the patient did not receive full treatment. The patient completed a total of 29 cycles of the Texas cocktail, alternating between partial response and stable disease throughout these 29 cycles. The last cycle was delayed by multiple weeks due to a dental infection. In August 2019 she presented to an outside emergency room complaining of back pain for the past 2 days, vomiting every day for the past few days, and pain in the right lower quadrant that had started that day. A non-contrast CT scan was performed.

\section{Dr Bhosale}

A non-contrast CT scan (August 2019) showed an increase in the liver metastases in segment VIII and the left lower quadrant implant. The liver metastasis was grossly similar. The left lower quadrant mass appeared larger measuring $5.9 \times 4.6 \mathrm{~cm}$ 


\section{Case study}

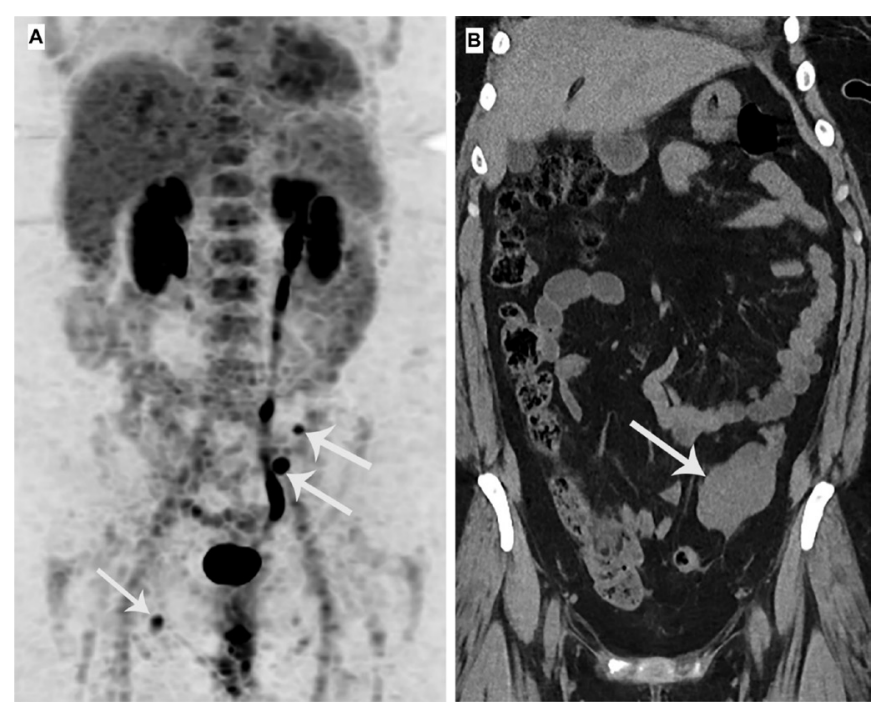

Figure 4 Maximum intensity projection image of the PET/ CT scan performed in June 2018 (A) shows implants in the left lower quadrant and the right inguinal region (arrows). Coronal non-contrast CT image (B) acquired in August 2019 shows the implant in the left lower quadrant has increased in size, suggesting progression of disease.

She was started on a phase $1 / /$ trial of topotecan with VX-970 (M6620), an ataxia telangiectasia and Rad3 related kinase inhibito,r from September to November 2019. A2 cycles, treatment was discontinued due to progression (Figure 4B).
After she progressed on the phase $\mathrm{I} / \mathrm{II}$ trial, the patient was recommended to start capecitabine $(2500 \mathrm{mg}$ per day by mouth) and temozolomide (340 mg per day by mouth). After four cycles a PET scan showed liver progression as well as retrocecal and pelvic nodes, a $6 \mathrm{~cm}$ left adnexal mass, and L4 vertebral body, and the decision was made for hospice care. After 53 months from initial diagnosis, the patient died of disease.

\section{Dr Frumovitz}

This case demonstrates the dire need for more therapeutic options for women with recurrent high-grade neuroendocrine cervical cancer. Currently, we have only two choices that have shown any activity - platinum and etoposide in the upfront setting and topotecan, paclitaxel, and bevacizumab in the recurrent setting. As high-grade neuroendocrine carcinoma of the cervix is an exceedingly rare disease, any trial will probably need to be multi-institutional with like-minded centers collaborating to accrue patients to studies.

Contributors GS: presenter; PR: pathologist; PB: radiologist; MF: discussant.

Funding Small/Large Cell Carcinoma of the Cervix: Sisters United; National Cancer Institute (P30CA016672).

Competing interests MF has research support from AstraZeneca and GlaxoSmithKline and is a speaker/consultant for Stryker.

Patient consent for publication Not required.

Provenance and peer review Not commissioned; externally peer reviewed. 